\title{
La « longue marche » d'un enseignement de l'histoire des sciences et des techniques
}

\section{Danielle Fauque}

\section{(2) OpenEdition \\ Journals}

Édition électronique

URL : http://journals.openedition.org/trema/83

DOI : 10.4000/trema.83

ISSN : 2107-0997

Éditeur

Faculté d'Éducation de l'université de Montpellier

Édition imprimée

Date de publication : 1 octobre 2006

Pagination : $34-47$

ISSN : 1167-315X

Référence électronique

Danielle Fauque, « La « longue marche » d'un enseignement de l'histoire des sciences et des

techniques », Tréma [En ligne], 26 | 2006, mis en ligne le 08 septembre 2010, consulté le 20 avril 2019.

URL : http://journals.openedition.org/trema/83 ; DOI : 10.4000/trema.83

Ce document a été généré automatiquement le 20 avril 2019

Trema 


\title{
La « longue marche » d'un enseignement de l'histoire des sciences et des techniques
}

\author{
Danielle Fauque
}

1 En 1996, Jean ROSMORDUC ouvrait les journées de Morgat par une réflexion sur la "longue marche" des historiens des sciences et des techniques. Ce stage faisait partie du programme national de la Direction des lycées et collèges du ministère de l'Éducation nationale. Il s'est déroulé à Morgat, dans le Finistère du 20 au 24 mai. J. ROSMORDUC pensait alors que le combat amorcé pour un enseignement de l'histoire des sciences et des techniques, depuis de nombreuses années, commençait à porter ses fruits (ROSMORDUC, 1997, p. 5). Aujourd'hui, un enseignement d'histoire des sciences et des techniques est assuré à l'université selon des dispositions qui dépendent des niveaux d'enseignement et des départements concernés. Il est aussi dispensé à l'IUFM en formation initiale pour les futurs enseignants. Les

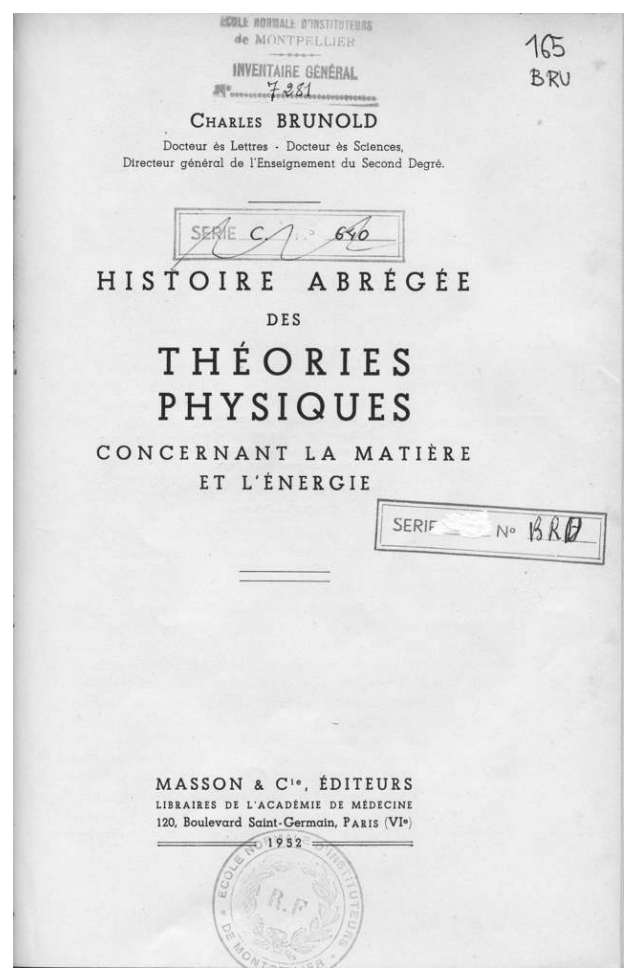
programmes scientifiques des lycées et des collèges, en particulier, font de plus en plus appel à l'histoire des sciences et des techniques. Pour arriver à ce succès, la marche a été effectivement longue. Sans remonter aux grands maîtres, comme Paul Langevin, qui déjà demandait qu'un tel enseignement soit constitué, penchons-nous seulement sur les vingt-cinq dernières années, une génération déjà. 


\section{La Société française d'histoire des sciences et des techniques (SFHST) et l'enseignement de l'histoire des sciences et des techniques (HST)}

2 Les origines de la SFHST remontent à l'entre-deux-guerres. Le comité national français, émanation de l'Académie internationale d'histoire des sciences fondée en 1928, a créé le Groupe français d'historiens des sciences en 1931 (TATON, 1980, p. 5). L'activité du groupe se poursuivit avec des fortunes diverses. Dans les années 1960, les bouleversements des structures universitaires, l'intérêt croissant porté à l'histoire des sciences et des techniques, sont des raisons impérieuses qui poussent le groupe à repenser les conditions de collaboration entre les différents membres de cette communauté. Il est alors décidé de créer une société française d'histoire des sciences et des techniques (SFHST) le 29 janvier 1980 (Id., p. 7).

3 Son premier président, Jacques ROGER, écrivait dans le premier bulletin : «Jamais, sans doute, l'histoire des sciences et des techniques n'a suscité autant d'intérêt en France » (ROGER, 1980, p. 1). L'envahissement de la science et de la technique, l'évolution de nos modes de pensée, le bouleversement épistémologique des sciences depuis cinquante ans, ont suscité cette demande. En conséquence, des groupes de recherches se sont formés en France pour étudier le phénomène scientifique et technique. Comme il y avait peu d'enseignements organisés, ces derniers étaient assurés principalement par des amateurs et des autodidactes en histoire des sciences. La discipline restait marginale en sciences et même en philosophie où elle était le mieux enracinée. Elle n'avait pas d'existence autonome ni au CNRS, ni dans les sections du CSCU. Sa situation était celle d'une discipline en plein développement intellectuel qui se heurtait à la pesanteur des mentalités, à la structure administrative où elle n'avait pas sa place. En conséquence, ajoutait J. ROGER, la SFHST se fixait comme objectifs d'informer les chercheurs de ce qui existait en France, de réunir ces chercheurs et ceux qui s'intéressaient à cette discipline, d'agir auprès du public cultivé, et auprès des pouvoirs en place, afin d'obtenir les moyens dont elle avait besoin. Puis le chercheur concluait : «L'histoire des sciences et des techniques, par la réflexion qu'elle permet sur les réalités de notre monde, doit être un élément essentiel de cette culture de demain » (ibidem).

4 La SFHST organise une première rencontre nationale à Nantes, les 9 et 10 octobre 1980, sur le thème de l'enseignement de l'histoire des sciences aux scientifiques (les techniques viendront plus tard). Il s'agit de faire le point sur l'enseignement donné à l'université comme option dans les DEUG scientifiques depuis trois ou quatre ans. Cet enseignement pose alors des problèmes de curriculum, d'organisation et d'articulation en amont (histoire des sciences dans le secondaire) et en aval (licence et maitrise, troisième cycle d'HS). On y souligne que les réformes de l'enseignement des années 60 gommaient l'appréciation du déroulement historique des disciplines (mathématiques et sciences de la vie et de la terre), que son impact social était ignoré. On observe aussi une volonté manifeste d'intégration de l'HST dans beaucoup d'établissements secondaires. Les instituteurs aussi y sont sensibles et demandent une formation en conséquence dans les nouveaux DEUG. Cet intérêt crée des besoins.

5 Ces différentes observations avaient donc conduit les organisateurs à présenter, au cours des journées de Nantes, un bilan de ce qui se faisait, une étude de quelques expériences 
étrangères, et à proposer de réfléchir ensemble sur les problèmes posés par les disciplines scientifiques, à des niveaux d'enseignement différents. Après les communications en séances plénières, des commissions indépendantes se sont réunies par niveaux d'enseignement, avec présentation d'expériences d'introduction de l'HS dans le secondaire.

6 Les actes furent édités rapidement par Jean DHOMBRES, avec un rapport général de J. ROSMORDUC sur l'enseignement en France de l'HS aux scientifiques. Ce dernier rappelait le rôle actif de R. TATON (rencontres, organisation de l'enseignement dans les DEUG, encouragements des expériences) et soulignait l'intérêt pédagogique de cet enseignement, observé par les collègues qui le pratiquent (meilleure compréhension de la discipline), l'intérêt méthodologique (la formation de l'esprit scientifique exige une réflexion sur les sciences et leur évolution, condition nécessaire mais non suffisante évidemment), l'intérêt idéologique (le rôle de la science et des techniques dans la société actuelle pose différents problèmes et fait débat). J. ROSMORDUC y ajoutait un intérêt, non relevé dans l'enquête, mais souligné par R. TATON, en 1965, au XIe Congrès international d'histoire des sciences (Varsovie, 1965), dans sa communication, «L'histoire des sciences et la science actuelle »: il était nécessaire que les décideurs connaissent aussi les principales tendances de l'évolution des sciences et les différents facteurs de la création scientifique (Delorme, 1966). Bien sûr, un enseignement séparé d'HST était inenvisageable jusqu'au baccalauréat. Il fallait donc s'orienter vers la formation des maîtres dans ce domaine (ROSMORDUC, 1980, p. 11). À l'université, un consensus se dégageait pour une nécessité de cet enseignement. Ces journées ont été suivies d'autres rencontres sur l'enseignement de l'HS dans les cursus de philosophie et d'histoire.

7 Au Congrès de la SFHST à Lille (2001), la session 1, consacrée à l'enseignement de l'histoire des sciences et des techniques, sous la responsabilité de Dominique LECOURT et de Véronique LE RU, était divisée en ateliers, dont un sur la formation des enseignants en HST. En marge, l'état des recherches dans ce domaine a été présenté dans un atelier autonome, sur l'histoire de l'enseignement des sciences, animé par N. Hulin et H. GISPERT (JOLY et JULLIEN, 2004, p. 47-52).

8 Au Congrès de Poitiers (2004), cette session a été reconduite sous le titre "Histoire des sciences et des techniques et enseignement" sous la responsabilité de Danielle FAUQUE et Hélène GISPERT. Le quatrième et dernier atelier, "Enseigner l'histoire des sciences aujourd'hui », s'est déroulé sous forme de deux tables rondes. D'une part H. GISPERT et M. SPRANZI-JOUBER, sous le titre «réalités institutionnelles, identités et enjeux disciplinaires ", prenaient en compte les résultats d'une vaste enquête menée depuis plus d'un an auprès des différents acteurs de l'enseignement de l'HS (présentation des premiers résultats à la journée d'étude du 20 juin 2003 à l'ENS - Ulm). D'autre part, une seconde table ronde réunissait des formateurs exerçant majoritairement en IUFM. Pour tous, l'enseignement de l'HST n'apparaît pas nécessaire pour enseigner les sciences, mais peut aider à comprendre la démarche scientifique. Pour les professeurs stagiaires, l'application directe en classe de l'enseignement reçu en IUFM est privilégiée. Pour les professeurs titulaires, elle est source d'un enrichissement personnel, elle permet de prendre du recul vis-à-vis du contenu disciplinaire. Mais on s'aperçoit que, dans les faits, l'HST est très peu utilisée en classe si ce n'est sous forme de dates et d'anecdotes. On est loin des idées des concepteurs des programmes.

9 Cette table ronde (la séance, très animée, s'est révélée trop courte en regard des enjeux révélés), qui a permis de faire émerger des besoins communs, a fait naître la nécessité 
d'approfondir le débat, et donc de se réunir à nouveau, ce qui fut fait à Montpellier les 20 et 21 mai 2005. Ce sont les actes de ces journées qui sont publiés ici.

\section{Enseignement de l'HST et formation des enseignants}

10 La nécessité de l'introduction de cet enseignement est apparue d'abord par la demande qui en a été faite. En particulier, plusieurs enseignants de sciences ont désiré mieux comprendre le processus de création et d'élaboration du fait scientifique. Cette demande a induit des actions à un plus haut niveau menée, entre autres, par des personnalités comme J. ROSMORDUC, alors enseignant à l'Université de Brest, et Hubert Gié, inspecteur général de sciences physiques.

11 J. ROSMORDUC a milité pendant de très nombreuses années pour cet enseignement en publiant des outils pour les enseignants, en organisant des universités d'été, des journées dans le cadre de plans académiques de formation et par ses nombreuses publications à but pédagogique ou de formation.

12 Dans ce contexte, les journées de Morgat ont constitué une des étapes les plus importantes d'un processus amorcé depuis 1980. La publication, qui a suivi, mêlant rapports d'expériences menées dans les classes à divers niveaux, études méthodologiques à destination des commençants en histoire des sciences et analyse de la présence de l'HST dans les programmes officiels, reste encore d'actualité aujourd'hui.

Depuis, de très nombreuses journées de formation ont été organisées dans les différentes académies avec des fortunes diverses. Corrélativement les IREM ont fourni un travail considérable pour la formation des maîtres en histoire des mathématiques, ils ont même abrité quelques formations en histoire des autres disciplines scientifiques. Évelyne BARBIN a témoigné abondamment de ces travaux. De nombreux enseignants se sont aussi lancés dans des travaux de thèse, dont le sujet était parfois allié à la didactique des disciplines. En particulier, cette formation est donnée au sein du Laboratoire interuniversitaire de recherche sur l'enseignement des sciences et des techniques (LIREST) depuis de nombreuses années.

14 Les besoins en formateurs en histoire des sciences et des techniques se sont accrûs avec la création des postes dits «Lecourt » à la fin des années 1990, sous le ministère de Claude Allègre. On se souvient aussi des grandes enquêtes lancées par ce ministre en 1998. Ainsi, la commission de chimie dirigée par Alain GAUDEMER, président de l'Université Paris XI Orsay, insistait-elle sur la dimension historique et culturelle de l'enseignement de la chimie. L'enquête menée auprès des professeurs de physique - chimie de onze académies révélait que l'histoire des sciences est une des disciplines nécessaires à la culture commune des élèves mais absentes de l'enseignement (GAUDEMER, 1998, p. 58). Le rapport de synthèse de la journée qui a clôturé les travaux de cette commission (Bordeaux, 1er avril 1998), souligne le rôle de l'HST dans la formation de l'élève, et ajoute que l'histoire des sciences et des techniques doit faire partie intégrante de la formation des maîtres (GAUDEMER, 1998, p.3). La commission recommandait aussi de créer des « travaux pluridisciplinaires encadrés » ou TPE, permettant l'interdisciplinarité et un réel travail personnel d'investissement de la part de l'élève, acteur de sa formation et de sa culture (Id., p.4). Cette activité permettrait aussi en particulier d'y introduire une dimension historique. Cette proposition de la commission de chimie fut retenue et a 
permis la naissance des «travaux personnels encadrés » ou TPE dont on sait le succès malgré les actions de nombreux détracteurs ; TPE qui ont finalement été supprimés en 2005 au niveau de la classe de terminale. Ils sont pour l'instant maintenus en classe de première.

Le rapport final, sous la présidence de Philippe MEIRIEU, objet du colloque de synthèse de Lyon, le 11 mai 1998, précise clairement que les disciplines scientifiques et technologiques contribuent à la culture commune (Id., p. 89), et souligne la nécessité d'une coopération entre les disciplines (Id., p. 94). Mais l'histoire des sciences et des techniques ne semble pas apparaître dans ce rapport. Cependant, depuis, l'institutionnalisation de l'enseignement de l'HST a avancée, ainsi que le développe $\mathrm{H}$. Gispert dans sa communication.

\section{Rencontres internationales}

16 Le fait que l'histoire des sciences et des techniques contribue à la culture moderne est accepté et défendu par un grand nombre de pays. Son enseignement et sa diffusion étaient déjà fortement recommandés dès la Libération. Dans la première conférence générale de l'UNESCO, en 1946, il a été souligné l'importance de l'histoire des sciences " projet d'importance cardinale pour l'histoire de la culture humaine » (CORTESAO, 1948, p. 29 ; FAUQUE, 2002, p. 53). L'œuvre de Paul TANNERY, l'activité de George SARTON, sont mises en avant. Pierre SERGESCU, historien des mathématiques, agit inlassablement pour promouvoir l'histoire des sciences au plan international en tant que telle, mais aussi pour sa diffusion avec la création des Conférences du Palais de la Découverte, à Paris. Sous son influence, l'AFAS, en France, crée une section d'histoire des sciences associée à la philosophie en 1945 (FAUQUE, 2002). Dans le cadre des manifestations organisées par cette société savante, au congrès de Caen en 1954, la section, sous la responsabilité de $R$. TATON, a pris pour thème "l'histoire de l'enseignement au XVIIIe siècle ", ouvrant un tout nouveau champ de recherches. L'ouvrage qui en est sorti reste aujourd'hui encore une référence (TATON, 1964). Dans le même temps en Grande-Bretagne, des discussions analogues se tenaient au sein de la British society for the history of science (BSHS) créée en 1947. Le problème de l'enseignement de l'histoire des sciences à l'école est soulevé à chaque rencontre (BJHS, 1997).

Les préoccupations des chercheurs français des années 1980 sont aussi celles des chercheurs étrangers. Sans être exhaustif, citons quelques actions. Celle de Fabio BEVILACQUA à Pavie tout d'abord. Professeur de physique à l'université A. VOLTA de cette ville, F. BEVILACQUA a développé des recherches et des formations d'enseignants des sciences en histoire des sciences et des techniques. Sous sa responsabilité, ont été publiés de nombreux documents pédagogiques et organisées de nombreuses rencontres dont les actes ont été publiés. Dès le début de son entreprise, il associe l'aspect historique des textes aux objets recueillis dans les musées ou reconstruits d'après la littérature de l'époque. Dès que des possibilités de diffusion et de production d'outils pédagogiques par l'informatique sont offertes, il utilise ce moyen pour produire des hypertextes interactifs portant sur l'histoire des sciences. En 1983, une première réunion internationale se tient à Pavie sur le thème "Using history of physics in innovatory physics education", coorganisée par F. BEVILACQUA et P. J. Kennedy de l'Université d'Édimbourg. L'esprit de ce colloque est bien dans la mouvance des idées de l'UNESCO et de l'IUHPS (Union internationale d'histoire et de philosophie des sciences), esprit repris par plusieurs 
groupes de recherches dans le monde, qui tend à promouvoir une nouvelle façon d'enseigner les sciences. On se réfère aux travaux de Georges HOLTON et au Project Physics Course (PPC), etc. Un public nombreux et venant de plusieurs continents vient partager ses expériences et débattre (BEVILACQUA, 1983). En 1986, les rencontres se poursuivent à Munich, au Deutsche Museum, pour le public européen cette fois (Thomsen, 1986), puis en 1988, à Paris, à la Cité des sciences et de l'industrie (BLONDEL et BROUZENG, 1988). Une dernière rencontre de ce type a lieu à Cambridge en 1990, mais n'a pas donné lieu à la publication d'actes. Au cours de ces différentes rencontres, la notion de "small injections " proposée par Silvana GALDABINI (Milan) rencontre un certain écho. Il ne s'agit pas d'organiser au niveau du secondaire un enseignement spécifique d'histoire des sciences et des techniques mais à l'occasion de telle ou telle notion d'en introduire des éléments. Cette proposition reflète bien le bilan des observations des enseignants qui avaient depuis plusieurs années déjà tenté d'introduire des aspects de l'histoire des sciences dans leur enseignement scientifique.

Depuis le début des années 1990, un grand nombre de pays a suscité des rencontres autour du thème de l'introduction d'une dimension historique dans les curricula. Les associations d'enseignants se sont montrées, un peu tardivement et encore timidement, favorables à cette demande. L'Union des Physiciens n'a accepté de publier un compte rendu d'une expérience présentée lors d'un stage en 1984 qu'en 1989, publication refusée en 1984 sous le prétexte qu'aucune demande n'avait été formulée sur ce sujet (FAUQUE, 1989). Mais en 1989, l'influence du professeur H. Gié se faisait nettement sentir, nous y reviendrons plus loin. Depuis cette date, le BUP a publié de nombreux articles d'information, de formation, de comptes rendus d'expérience, de méthodologie en enseignement de l'histoire des sciences. Les sections locales de l'association ont organisé des demi - journées de stage consacrées à l'histoire des sciences et des techniques tout au long des années 1990.

En Italie, l'Associazione per l'insegnamento della fisica (AIF) a consacré un numéro de son périodique trimestriel à la place que l'histoire de la physique occupe dans la didactique de la physique (AIF, 1995). En particulier, Fabio BEVILACQUa y présente l'expérience européenne alliant didactique et histoire que nous avons évoqué ci-dessus. Les articles de ce numéro sont écrits par des auteurs collaborant à son groupe de recherche, et qui sont souvent intervenus dans les rencontres internationales. Y participe aussi Michael R. MATTHEWS (University of New South Wales, Sydney, Australie), qui vient à ce moment de fonder une revue spécialisée, Science and Education. En Grande-Bretagne, Michael SHORTLAND et Andrew WARWICK avaient publié Teaching the History of Science, sous l'égide de la BSHS, en 1989. Cet ouvrage inscrit aussi dans l'histoire le processus d'intégration de l'idée d'introduction d'une dimension historique dans l'enseignement des sciences, à la fois dans la formation des enseignants et dans celle des élèves.

En 1998, Claude DEBRU réunissait à Strasbourg, un grand nombre de chercheurs et d'enseignants. Ce congrès était organisé conjointement par l'université Louis PASTEUR et la division d'histoire des sciences de l'Union internationale, sous l'égide de l'union des académies européennes (All european academies - ALLEA), sur une proposition de Paul GERMAIN, de l'Académie des sciences. Il a donné lieu à une publication par la commission européenne en 1999 (DEBRU, 1999). Ce panorama très varié de l'enseignement de l'histoire des sciences dans le monde permet de se rendre compte de la place que prend l'HST dans la société actuelle, mais a laissé plusieurs participants sur leur faim. Trop de rapports n'exposent que des intentions ou des considérations très générales, peu 
témoignent d'une véritable réflexion née d'une pratique effective de cet enseignement en particulier aux niveaux précédents l'université. L'enseignement de l'histoire des sciences y apparaît déjà comme assez académique et la discipline trop centrée sur elle-même. En allant plus loin, il apparaît que l'enseignement des sciences est lui-même à transformer. Plaquer un enseignement traditionnel d'histoire des sciences à côté d'un enseignement traditionnel des sciences ne changerait probablement rien quant à la compréhension du phénomène scientifique et de son rôle dans la société d'aujourd'hui. Néanmoins, cette rencontre a permis de souligner l'unité des questionnements dans le monde occidental, questionnements dont les réponses tardent souvent, faute de moyens et peut-être de volonté politique, à émerger. Cette publication présente aussi un rapport des formes des différents enseignements d'histoire des sciences, dispensés en Europe.

Dans la suite du congrès de Strasbourg, la BSHS, sous l'égide de la Royal Society, a organisé en juillet 2000 à Londres une rencontre autour de Science communication, education and the history of science (www.ucl.ac.ul/sts/bshs/abstract/6-5.htm). Lors de ce colloque, des sujets classiques ont été débattus : rôle de l'histoire des sciences dans la société, forme de la diffusion et de l'histoire des sciences par le musée, les expositions, les publications, les émissions télévisées, puis plus particulièrement l'histoire des sciences dans l'enseignement, à travers plusieurs exemples européens.

La commission européenne chargée des affaires culturelles a développé un programme de subventions pour les universités qui favoriseraient un projet international de formation des professeurs en histoire des sciences afin qu'ils introduisent cette dimension dans leur enseignement (Actions Comenius 2-1 et 3-1 du programme SOCRATES). C'est ainsi que deux projets ont vu le jour à peu près en même temps, le projet de l'université de Hull en Grande-Bretagne (HST Project), Teaching and learning the history of European science and technology, dirigé par Bert Sorsby de 1998 à 2002, qui a donné lieu à publication et à la création d'un site Internet, Merlin, et le projet Pénélope (the Penelope project), dirigé par John CARTWRIGHT (Chester College of Higher Education). Ces projets ont conduit à la réalisation d'un stage suivi par des enseignants européens. Le projet Hull a permis un travail interdisciplinaire au sein du groupe français formé par des enseignants de l'école primaire, du collège et du lycée, sur des exemples d'application effective d'activités à trame historique en classe, dans différentes disciplines. Le travail international a aussi permis de mieux se connaitre entre nations, de mieux se comprendre, et a permis aussi de découvrir dans quelles conditions l'enseignement à l'école primaire était dispensé en Norvège, en Irlande, en Angleterre, au Portugal ou en France, et comment à chaque niveau, dans chaque pays, un peu d'histoire des sciences était dispensé. Mais l'ambition de cet enseignement était extrêmement humble. Une compréhension commune de l'importance des sciences et des techniques et de la nécessité d'en enseigner des éléments d'évolution reste l'apport principal de ces ambitieux projets (SORSBY, $2002 \mathrm{abc}$ ). Ils peuvent aussi donner, et dans notre cas ont conforté, l'idée d'une Europe à l'héritage commun qu'il faut transmettre. 


\section{IV. Évolution de la présence de l'HST dans les programmes officiels de l'enseignement secondaire général, dans le cas particulier des sciences physiques}

Une grande réforme des lycées (résultant des travaux de la commission LAGARRIGUE) propose en 1979 pour la première fois de faire appel à l'histoire des sciences et des techniques dans l'enseignement des sciences physiques aux classes littéraires. Cette phrase va permettre d'ouvrir un champ d'expériences dont les comptes rendus seront communiqués au cours de rencontres nationales ou internationales, et publiés dans les divers actes. Engagée avec prudence, ces tentatives ont été particulièrement observées par H. GIE. Elles ont donné lieu à des débats, des discussions, des recommandations, mais aussi à un encouragement continu et fort. Les résultats de ces expériences ont été pris en compte lors de la rédaction des programmes de 1993, rédaction dans laquelle H. GIE a joué un rôle important. La structure de l'enseignement des sciences physiques évolue vers un ensemble thématique, plus accentué en chimie qu'en physique. Le document d'accompagnement du programme de physique - chimie de la classe de terminale scientifique présente les aspects historiques mis explicitement au programme de mécanique et d'optique, et propose une bibliographie assez large même si elle est limitée aux thèmes abordés en terminale (MEN, 1995, pp. 28-48). Aux journées de Morgat, H. GIE soulignait lui-même le rôle de l'histoire des sciences dans les nouveaux programmes des filières scientifiques du secondaire : «Il importe de faire comprendre, autant que possible, comment se construit la science avec ses hésitations, ses longues périodes de réflexion, ses allers et retours, et de montrer la difficulté d'accéder aux concepts nouveaux, même chez les plus grands esprits. L'attitude scientifique consiste à questionner, à remettre en cause, et à abandonner parfois de grandes certitudes. Le progrès se construit sur ce questionnement continuel de la nature par la mise en œuvre d'expériences appropriées qui infirment ou confirment les idées en cours. C'est donc là une démarche faite d'humilité constructive sur laquelle s'élève petit à petit l'échafaudage scientifique, le verdict expérimental restant toujours la référence » (GIE, 1997, p. 81). H. GIE présente ensuite quelques recommandations : choisir des exemples significatifs, ne pas négliger la chronologie, faire en sorte de citer tout au long de son enseignement les noms des auteurs des découvertes scientifiques, et la chronologie succincte les concernant, procéder à l'examen de textes scientifiques originaux ou de seconde main, guider les élèves par des questions appropriées (Id., p. 82). Il recommande aussi la lecture attentive du document d'accompagnement qui propose une petite initiation à l'histoire des sciences pour les professeurs, qui sont le plus souvent novices dans le domaine. On y souligne l'évolution non linéaire des idées et le rôle de l'expérience dans cette évolution. Le hasard peut intervenir, mais il ne suffit pas. Il faut un regard préparé à voir. Plus largement l'observation ne précède pas nécessairement la théorie. Cette dernière détermine souvent les expériences à mener (Id., p. 83). Ce texte d'H. Gié est toujours d'actualité.

On peut donc dire qu'avec ces nouveaux programmes, l'histoire des sciences et des techniques fait une apparition officielle en enseignement scientifique pour les élèves de 
section scientifique. Cette tendance va s'accentuer au cours des réformes suivantes, entraînant par là - même la formation initiale et continue des enseignants (MEN, 2000).

Dans les nouveaux programmes de cinquième et quatrième parus au mois d'août 2005, l'appel à l'histoire des sciences et des techniques est clairement présent dans le contenu des programmes et dans les recommandations, et plus particulièrement dans les thèmes de convergence (BO, 2005).

Parallèlement à l'évolution des programmes en faveur d'une plus grande introduction de l'histoire des sciences dans les programmes, deux actions ont été soutenues par l'Éducation nationale. En 1996, un groupe d'enseignants s'est réuni autour de l'inspecteur général Jean-Michel BERARD, pour étudier la forme que pourrait prendre un ouvrage proposant des études documentaires pour les élèves, ouvrage destiné aux enseignants. L'histoire des sciences peut être l'occasion de cette activité documentaire. C'est ainsi que l'ouvrage, La gravitation, est né et a été publié par le CNDP en 1997 (SONNEVILLE et FAUQUE, 1997). Il relie histoire des sciences et documentation plus contemporaine autour du thème de la gravitation sous forme de textes commentés et de questionnaires destinés aux élèves. De son côté, l'INRP publiait les résultats d'un travail interdisciplinaire dirigé par Frédéric AUDIGIER et Pierre FILLON, Enseigner l'histoire des sciences et des techniques, en 1991. C'est dans le cadre des activités du département « didactique des disciplines » que ce travail a été fait.

Mais quelle est l'attitude des didacticiens vis-à-vis de l'histoire des sciences ? Un livre vient de paraître, dirigé par Monique GOFFARD et Annick WEIL-BARAIS, Enseigner et apprendre les sciences (A. COLIN), qui présente les recherches et travaux faits au LIREST, replacés dans une perspective historique d'évolution de la didactique. Les chemins suivis par les historiens des sciences soucieux d'introduire un enseignement d'histoire des sciences et des techniques, et les chemins des didacticiens soucieux de mieux comprendre l'acte d'apprendre les sciences ont évolué parallèlement, et pourtant ont des racines communes, nées des recherches effectuées dans les années 1960 principalement aux États-Unis et en Angleterre, respectivement dans les projets PSSC (Physical Science Study Committee), HPP (Harvard Project Physics) et Nuffield en Angleterre (BELHOSTE, GISPERT, HULIN, 1996). Il s'agit maintenant de rendre l'élève acteur de sa formation, en éveillant son esprit d'investigation et en développant une pensée créative et raisonnée (projet NUFFIELD). Il faut donc revoir les curricula ; l'histoire des sciences peut répondre à une partie des problèmes (comment s'élabore le processus de création), l'étude du processus d'apprentissage peut contribuer à réformer l'enseignement des sciences. Le PSSC a publié des textes, traduits en plusieurs langues, qui ont servi de départ à de multiples recherches, en enseignement de l'histoire des sciences et en didactique. Ce que beaucoup ont oublié, c'est que les propositions de ces projets reposent sur une méthode plus ancienne, largement diffusée à la fin du XIXe siècle en Angleterre, la méthode heuristique du professeur ARMSTRONG, qui a dépassé largement le cadre de l'enseignement des sciences physiques et a été utilisée en particulier dans la toute nouvelle science de l'éducation (the science of teaching) à la fin du XIXe siècle. Armstrong avait rapporté les bases de cet enseignement d'Allemagne où il avait été parfaire sa formation de chimiste. Il avait été enthousiasmé par les méthodes d'enseignement des sciences utilisées outre-Rhin. Mais ceci est une autre histoire.

La structure des publications du HPP reste intéressante pour les formateurs des maitres en histoire des sciences, aujourd'hui. Une traduction canadienne diffusée par VUIBERT 
propose un enseignement de la physique entièrement basé sur une approche historique des concepts (IPR, $1980 \mathrm{abcd}$ ).

Aujourd'hui, les didacticiens et les enseignants en histoire des sciences et des techniques en IUFM se retrouvent devant la même tâche : former des enseignants en sciences. Les uns autant que les autres puisent dans les fonds des uns et des autres de nouvelles propositions. Les journées qui s'ouvrent à Montpellier permettront de mieux définir les enjeux et de faire avancer le débat.

\section{Conclusion}

Cette première rencontre à Montpellier va en appeler d'autres. Un groupe spécifique se forme. L'enseignement de l'HST et la formation des enseignants, initiale et continue, constituent une spécificité dans la discipline HST, qui désire aujourd'hui définir ses buts et ses méthodes et s'enraciner dans une histoire. Elle en a une, encore largement ignorée des protagonistes qui souvent refont le même chemin de découvertes et d'essais que les anciens. Cette histoire est construite par des textes nombreux qui ne sont pas assez diffusés. Trop de personnes se plaignent encore de n'avoir pas de textes de référence à consulter. La constitution d'un groupe spécifique des formateurs en HST en IUFM, aux objectifs clairement définis et revendiqués, permettrait d'avancer dans la voie d'une reconnaissance plus forte de la part de l'Éducation nationale.

Je termine ici cet exposé qui résume les points essentiels de l'historique de l'enseignement de l'histoire des sciences et des techniques dans les classes prébaccalauréat et dans la formation de leurs enseignants. Souhaitons qu'il soit utile à tous ceux qui désireront en explorer des aspects plus spécifiques.

\section{BIBLIOGRAPHIE}

Associazione per l'insegnamento della fisica (AIF). 1995. "La storia della fisica nella didattica della fisica", La fisica nella scuola, quaderno 5, anno XXVIII, al. $\mathrm{N}^{\circ} 2$, aprile-giugno.

AUDIGIER, Frédéric, FILLON, Pierre. 1991. Enseigner l'histoire des sciences et des techniques. Paris, INRP.

BELHOSTE, Bruno, GISPERT, Hélène, HULIN, Nicole (dir.). 1996. Les sciences au lycée. Un siècle de réformes des mathématiques et de la physique en France et à l'étranger. Paris, Vuibert \& INRP.

BERNARD, Maurice. 1997. "L'histoire dans l'enseignement des sciences physiques", Bulletin de l'Union des physiciens (BUP), nº 796, pp. 1433-1436.

BEVILACQUA, Fabio, KENNEDY, P. J. (ed.). 1983. Proceedings of the international conference on using history of physics in innovatory physics education, Pavie, 5-9 September 1983. Pavie, sd.

BLONDEL, Christine, BROUZENG, Paul (dir.). 1988. Science education and the history of physics / Enseignement scientifique et histoire de la physique, Paris, 21-25 novembre 1988. Paris, Cité des sciences et de l'industrie, Orsay, Université Paris-XI. 
BRAM, Georges, CHAMOZZI, Françoise, FUCHS, Alain, GRELON, André, LANCIANO-MORANDAT, Caroline, MORDENTI, Laurence (éd.). 1995. La chimie dans la société, actes du colloque de Biarritz, 23-25 mars 1994. Paris, L'Harmattan.

British Journal for the History of Science (BJHS), March 1997, special issue, vol. 30, part. I, n 104.

British Society for the History of Science (BSHS). (2000). Science communication, education and the history of science, conference, Londres, 12-13 juillet 2000. www.bshs.org.uk.

Bulletin officiel de l'Éducation nationale (BO). 2005. Programmes des collèges, thèmes de convergence, $\mathrm{n}^{\circ} 5$, hors série, 25 août 2005, pp. 61-67.

CORTESAO, Armando. 1948. "L'UNESCO, sa tâche et son but concernant les sciences et leur développement historique", Actes du Ve congrès international d'histoire des sciences, Lausanne 1947, pp. 25-35. Paris, Hermann.

DEBRU, Claude (ed.). 1999. History of science and technology in education and training in Europe, actes du congrès ALLEA (all european academies), Strasbourg, 25-26 juin 1998. Luxembourg, Office for Official Publications of the European Communities.

DELORME, Suzanne. 1966. "Le XIe Congrès international d'histoire des sciences, Varsovie-Cracovie, août 1965", Revue d'histoire des sciences, XIX, pp. 59-63.

DÉSAUTELS, Jacques, ANADON, Marta, LAROCHELLE, Marie. 1988. Le culte de la science, les programmes d'enseignement des sciences en question. Québec, Ottawa, laboratoire de recherches sociologiques, département de sociologie, Université Laval.

DHOMBRES, Jean (dir.). 1980. Actes du colloque Enseignement de l'histoire des sciences aux scientifiques, Nantes, 9-11 octobre 1980. SFHST \& Université de Nantes.

FAUQUE, Danielle. 1989. "L'enseignement de l'histoire des sciences dans les classes du secondaire", Bulletin de l'Union des physiciens (BUP), $\mathrm{n}^{\circ}$ 712, pp. 417-424.

FAUQUE, Danielle. 1998. "La dimension historique dans l'enseignement scientifique secondaire en France", Bulletin de l'Union des physiciens (BUP), n 803, avril 1998, pp. 623-636.

FAUQUE, Danielle. 2002. "L'histoire des sciences au sein d'une société savante, l'Association française pour l'avancement des sciences (1945-1978)", Archives internationales d'histoire des sciences, vol. 52, pp. 46-67.

FRAGA, Xosé A. (ed.). 1997. Ciencias, educación e historia. Actas V Simposio de Historia e Ensino das Ciencias, Vigo, 1995. Sada, La Coruña, Galice, Publicacións do Seminario de Estudos Galegos, Edicios do Castro.

GAUDEMER, Alain (dir.). 1998. Quels savoirs enseigner dans les lycées ?, journée disciplinaire sur l'enseignement de la chimie, Bordeaux, 1er avril 1998. Orsay, édition de l'université.

GIÉ, Hubert. 1997. "L'histoire des sciences dans les nouveaux programmes des filières scientifiques du secondaire", in ROSMORDUC (1997), pp. 81-88.

GOFFARD, Monique, WEIL-BARAIS, Annick (dir.). 2005. Enseigner et apprendre les sciences, recherches et pratiques. Paris, A. Colin.

GOTT, Richard, DUGGAN, Sandra. 1995. Investigative work in the science curriculum. Buckingham, Philadelphia, Open University Press.

GUEDJ, Muriel, DUSSEAU, Jean-Michel. 1999. "À propos d'une formation des enseignants de sciences physiques à l'épistémologie et à l'histoire des sciences", Bulletin de l'union des physiciens (BUP), $\mathrm{n}^{\circ}$ 815, cahier $n^{\circ} 1$, pp. 991-1005. 
Institut de recherches psychologiques (IPR). 1980a. Série Harvard Project Physics, t. I : Les concepts de mouvement.-1980b. t. II : Les mouvements célestes.-1980c. t. III :Le triomphe de la mécanique. 1980d. - t. IV : La lumière et l'électromagnétisme. Traduction française du HPP. Montréal, Institut de recherches psychologiques. Diffusion Vuibert.

JOLY, Bernard (dir.). 2004. Actes du Congrès d'histoire des sciences et des techniques, Poitiers, 2004. Cahiers d'histoire et de philosophie des sciences, hors série. Lille, Imprimerie de l'université (à paraître).

JOLY, Bernard, JULLIEN, Vincent (dir.). 2004. Actes du Congrès d'histoire des sciences et des techniques, Lille, 2001. Cahiers d'histoire et de philosophie des sciences, hors série. Lille, Imprimerie de l'université.

JULIA, Bernard (dir.). 1991. Les objectifs de la formation scientifique, Actes du colloque, École polytechnique, Palaiseau, 28-29 avril 1990. Nancy, imprimerie Bialec.

LEVINSON, Ralph (ed.). 1995. Teaching science. London, New York, Routledge \& The Open University.

MATTHEWS, Michael R. (ed.). 1992a. "Editorial", Science \& Education, vol. 1, n 1, pp. 1-9.- 1992b. "History, philosophy and science teaching : the present rapprochement". Science \& Education, vol. 1, $\mathrm{n}^{\circ}$ 1, pp. 11-47. Kluwer Academic Publishers, Dordrecht, Boston, London.

Ministère de l'éducation nationale (MEN). 1995. Document d'accompagnement du programme de physique-chimie de la classe de terminale scientifique (enseignement obligatoire), part. I.

Ministère de l'éducation nationale (MEN). 2000. Document d'accompagnement du programme de physique-chimie de la classe de seconde, partie chimie.

ROGER, Jacques. 1980. "Préface", Bulletin de la SFHST, n¹, p. 1.

ROSMORDUC, Jean. 1980. "L'enseignement en France de l'histoire des sciences aux scientifiques", in DHOMBRES, Jean (1980), pp. 5-15.

ROSMORDUC, Jean (dir.). 1997. Histoire des sciences et des techniques. Actes des journées de Morgat. Rennes, CRDP de Bretagne.

SHEIDECKER, Myriam, LAPORTE, Gérard. 1998. "À propos du débat sur "l'histoire dans l'enseignement des sciences physiques"', Bulletin de l'Union des physiciens BUP, n803, avril 1998, pp. 637-639. SHORTLAND, Michael, WARWICK, Andrew (ed.).1989. Teaching the history of science. Blackwell, Oxford.

SONNEVILLE, Madeleine, FAUQUE, Danielle. 1997. La gravitation. Paris, CNDP.

SORSBY, Bert (ed.). 2002a. Teaching and learning the history of European science and technology. 2002b. Teaching and learning the history of European science and technology : Ressource manual. - 2002c. Teaching and learning the history of European science and technology : Training manual. Bucarest, Ars Docendi.

TATON, René (dir.). 1964. Enseignement et diffusion des sciences au XVIIIe siècle. Paris, Hermann. Rééd. 1986.

TATON, René. 1980. "Sur les origines de la SFHST", Bulletin de la SFHST, n¹, pp. 5-7.

The Project Physics Course. 1971. New York, Toronto, Holt, Rinehart and Winston, Inc.

THOMSEN, Poul V. (ed.). 1986. Proceedings of the multinational teacher / teacher trainer Conference on Science Education and the History of Physics, Munich, 3-9 May 1986. Aarhus, DK, université d'Aarhus. 


\section{RÉSUMÉS}

Dès sa fondation (1980), la SFHST a milité en faveur d'un enseignement de l'histoire des sciences et des techniques. Jean Rosmorduc et Hubert Gié ont fait beaucoup en ce sens pour la formation des enseignants. Cette action s'inscrit aussi dans un vaste mouvement international qui du congrès de Pavie (1983) à la rencontre de Londres (2000) montre le rôle croissant de cet enseignement. Les futurs programmes de sciences au collège l'introduisent encore plus clairement, dans un projet interdisciplinaire. Cet article voudrait retracer l'historique de cette action.

Since its foundation (1980), the SFHST has argued in favour of a teaching in history of science and technology. Jean Rosmorduc and Hubert Gié have made a lot in this sense for the training of the teachers. This action is lying within a vast international movement, which, from the congress of Pavia (1983) to the London meeting (2000), reveal the rising role of this teaching. The future science schedules of the collège (12-15) still place this one more clearly, within an interdisciplinary project. This paper would try to relate the history of this action.

\section{INDEX}

Mots-clés : enseignement, formation des maîtres, histoire des sciences

\section{AUTEUR}

\section{DANIELLE FAUQUE}

Société d'histoire et des techniques SFHST - Groupe d'histoire et de diffusion des sciences d'Orsay GHDSO danielle.fauque@stanislas.fr 